\title{
Evaluation and operative technique to repair isolated sternal fractures
}

\author{
J. Ernesto Molina, MD, PhD
}

From the Department of Surgery, Division of Cardiovascular and Thoracic Surgery, University of Minnesota, Minneapolis, Minn.

Received for publication Nov 23, 2004; revisions received Jan 27, 2005; accepted for publication Feb 16, 2005.

Address for reprints: Ernesto Molina, MD, Department of Surgery, Division of Cardiovascular and Thoracic Surgery, University of Minnesota, 420 Delaware St E, MMC 182, Minneapolis, MN 55455 (E-mail: molin001@umn.edu).

J Thorac Cardiovasc Surg 2005;130:445-8

$0022-5223 / \$ 30.00$

Copyright $\odot 2005$ by The American Association for Thoracic Surgery

doi:10.1016/j.jtcvs.2005.02.022
A series of 12 patients with traumatic sternal fractures without internal organ injury are reported to have had their fractures repaired with a standard operative technique that has proved to be effective up to 17 years of follow-up. Although sternal fractures are often seen in emergency departments of trauma centers, rarely are they recommended for correction. Often patients experience physical deformity, chronic pain, and abnormal physical habits. A standard operative technique is proposed with highly satisfactory results. Thoracic surgeons should be familiar with this problem and with available standard methods to correct it.

$\mathrm{S}$ ternal fractures are uncommon. They are usually caused by severe trauma as a result of falls, ${ }^{1}$ direct blunt trauma to the sternum, or, most frequently, car accidents. $^{2-6}$ The use of restraining seat belts apparently has increased the incidence of sternal fractures. ${ }^{3,5}$ Previous reports in the past 50 years described their incidence and the low mortality rate when no internal injuries occur, ${ }^{6,7}$ yet no reports have established a proper operation to repair them when obvious deformities or symptoms exist. Most of the time, sternal fractures are not treated directly: only conservative observation has been recommended. ${ }^{4,7}$

Of all the admissions recorded in emergency departments, sternal fractures account for $0.45 \%$ to $4 \%{ }^{4,6}$ The morbidity and mortality of such fractures are directly related to the extent of concomitant injuries and not specifically to the broken sternum. ${ }^{3,8}$ More than $95 \%$ of patients with a fractured sternum are treated conservatively. No reports are available on the late sequelae or evolution of such fractures. Recently, only $1.7 \%$ of the patients described by Potaris and associates ${ }^{4}$ were recommended for surgical intervention, and no specific operative technique was proposed.

The reason for not correcting sternal fractures in a standard manner is multifactorial: the lack of a standard approach to deal with this problem, the lack of experience among surgeons for implementing any specific operative technique, and, mostly, the lack of proper evaluation of the degree of deformity and of the long-term sequelae. We contend that many sternal fractures need to be surgically corrected to reestablish the proper orientation of the sternum, to avoid physical deformity, to prevent the slouch position that patients later experience, and to prevent chronic pain often triggered by any physical activity with the arms.

\section{Materials and Methods}

At our institution, we saw 12 consecutive patients with isolated sternal fractures from January 1987 through June 2004. All of the fractures resulted from severe trauma. The mean patient age was 32 years (range, 17-44 years). The group comprised 9 male and 3 female subjects. Only 2 of the 12 patients were seen acutely immediately after the trauma occurred. The other 10 patients came late to our attention, after they had been seen in other hospitals and discharged to follow no specific treatment other than pain relief in connection with any physical activity. All 12 patients demonstrated obvious sternal deformities, with a bulge at the site of the fracture. All 12 also exhibited a hunched posture, restricted neck extension, and kyphosis. The 2 patients we saw after the acute event had no other internal injury of the chest 

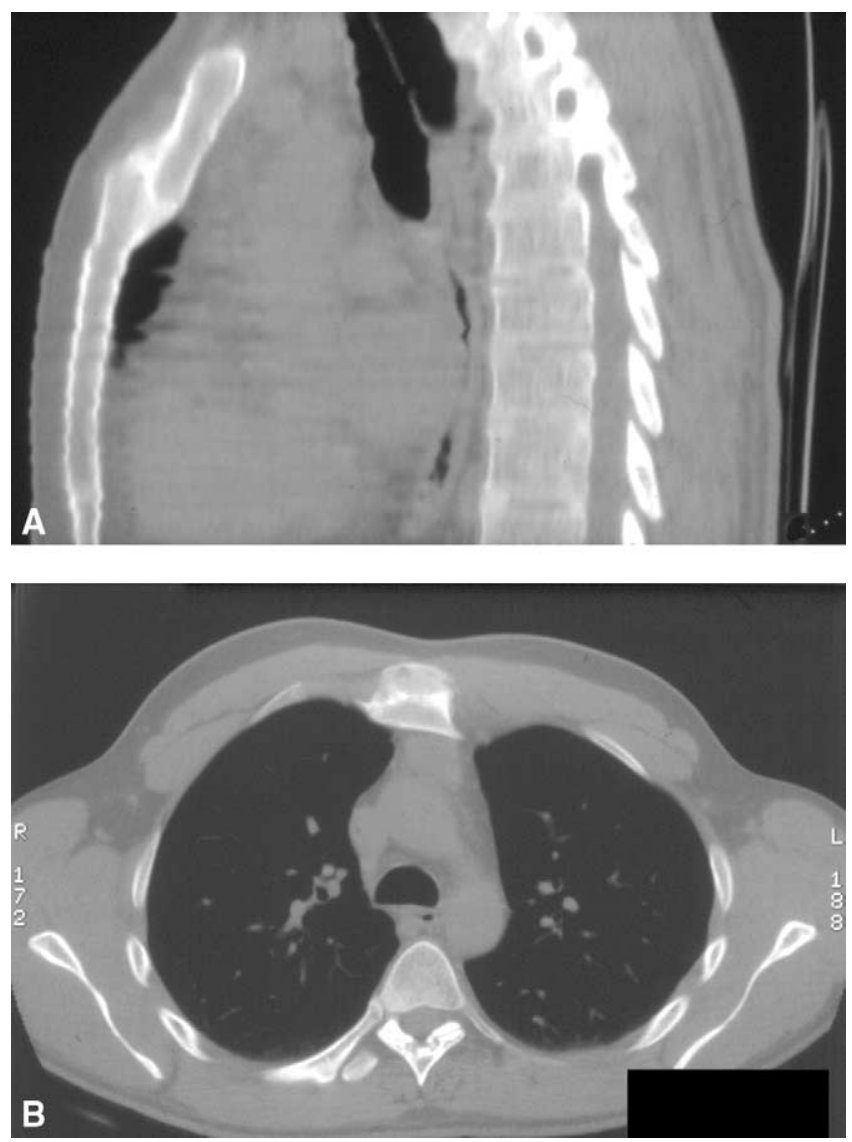

Figure 1. A, Computerized scanogram, lateral view, of the sternum showing the displacement of the manubrium behind the body of the sternum. B, Cross-sectional view of the sternum, with the distal body of the sternum anteriorly and the proximal manubrium behind posteriorly.

organs. The 10 patients we first saw in the chronic state also had no other internal abnormalities.

\section{Age of the Fracture}

Of the 12 patients we saw, 10 were already in the chronic stage (mean, 1.9 years after the original trauma; range, 1 month to 5 years). All fractures occurred in a transverse fashion; 1 of them had partially healed but with the unstable mobility typical of a nonunion fracture.

\section{Radiologic Evaluation}

The plain chest radiograph was useful to show the level of the fracture, ${ }^{9}$ but computed axial tomographic scan with tridimensional reconstruction was the most useful test for assessing the position of the fragments and the degree of overlapping in the 2 fragments of the sternum. It is important to note that all 12 patients did have overlapping of the bone fragments: the manubrium portion of the sternum was

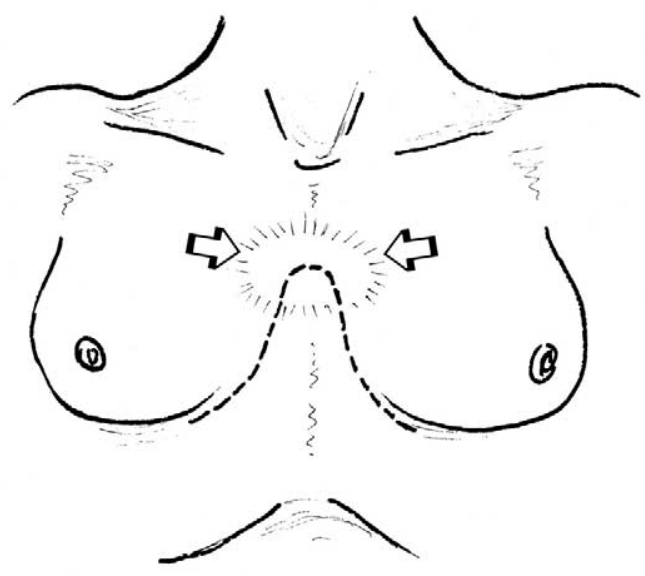

Figure 2. Surgical incision used to approach the sternal fracture having the highest curvature of incision over the protruding deformity.

displaced behind the body of the sternum, with an overlap distance of 1 to $3 \mathrm{~cm}$ (Figure 1). Externally, such a deformity might not be very obvious in an obese person, but a slender patient has a significant obvious deformity (in addition to all the symptoms that accompany this fracture). All 12 of our patients had the sternal fracture in the upper third of the sternum. For most of them, the first rib stayed attached to the upper sternal portion of the bone overlapping behind the body of the sternum.

\section{Surgical Approach}

Our incision is a bilateral subthoracic type that extends superiorly to the level of the fracture, where the 2 incisions are joined (Figure 2). In women the subthoracic group is easier to delineate than in men, but the same approach is used for all patients. Flaps are created superiorly and inferiorly to expose the entire length of the sternum. The pectoralis major muscles are not entered in the same direction but vertically, and they are undermined laterally to 4 or $5 \mathrm{~cm}$ away from the midline.

Once the level of the fracture is identified, we use cautery to dissect all the fibrous tissue formed between the 2 fragments of the bone until the 2 halves are freed up completely (Figure 3). A bone grasper is used to hold the upper fragment of sternum, and a second bone grasper is used to fasten the lower body of the sternum or distal portion. Occasionally, the internal thoracic vessels need to be ligated. Using an osteotome, we create a groove along the broken width of the distal end in the body of the sternum and make a bevel-tapered edge in the proximal broken bone fragment. Once this is accomplished, we overextend the operating table to create a slight opisthotonos of the chest that allows us to exert traction on both fragments of the sternum until 


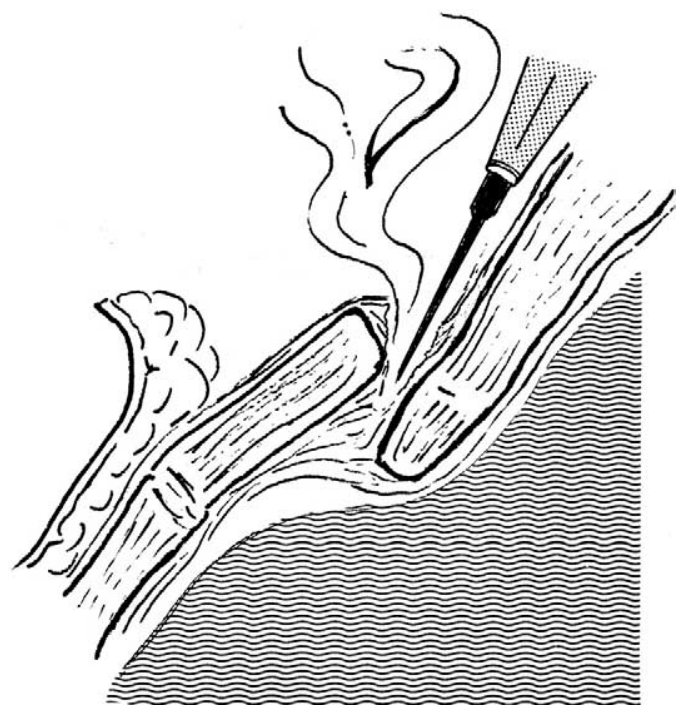

Figure 3. Detachment of the 2 fragments of the sternum using mostly the cautery until the 2 fragments are freed up.

they engage and lock each other at the prepared fracture level. Using a hand drill, we then make 2 holes in the upper portion of the sternum, as well as in the distal bone for future placement of sternal wires (Figure 4). Figure 5 shows the position of the sternal wires. We prefer to use gauge 6

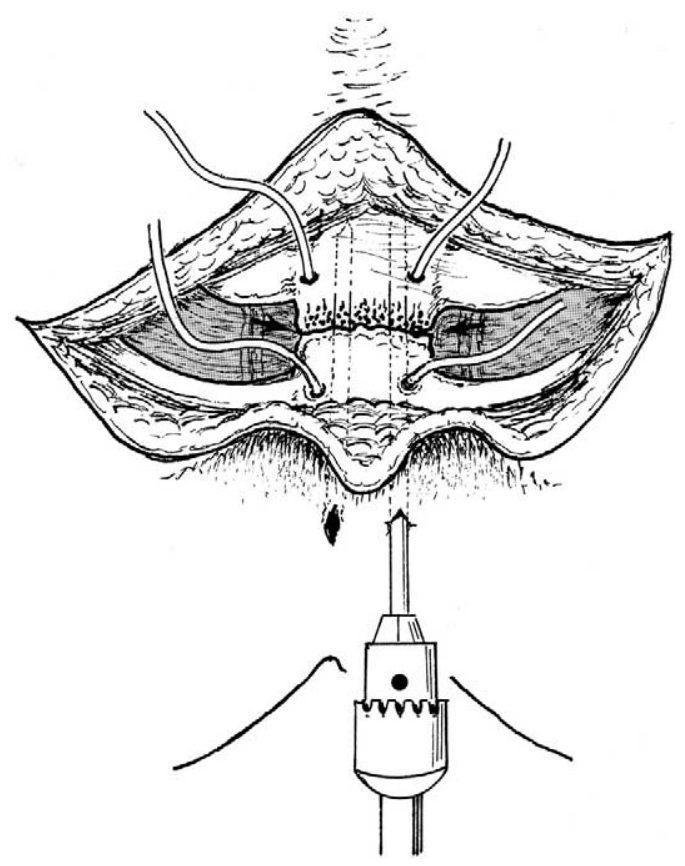

Figure 4. Two threaded Steinmann pins parallel to each other are inserted tangentially within the thickness of the distal fragment advanced across the fracture into the manubrium of the sternum.

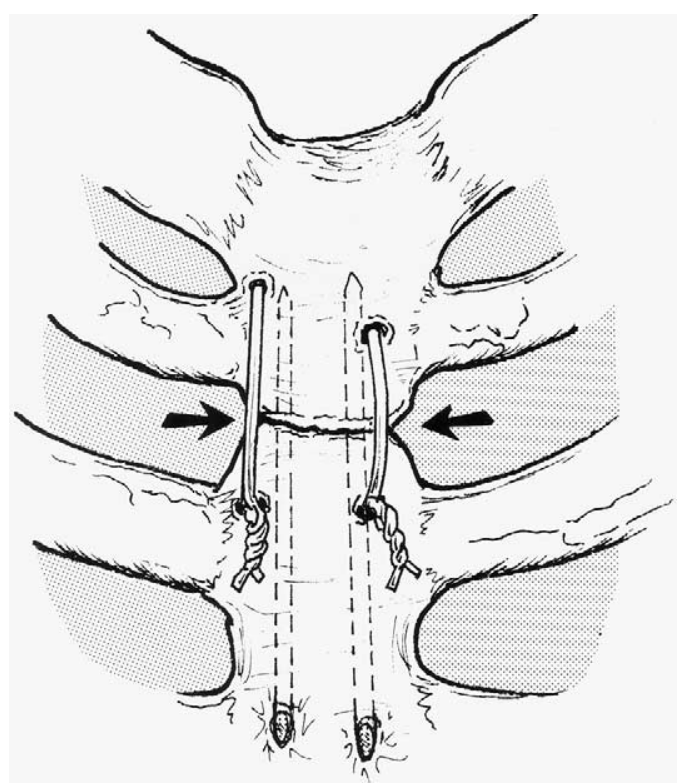

Figure 5. Complete repair with the pins in their normal position and the wires twisted together to prevent separation of the fragments.

or 8 , depending on the size of the patient. The wires are not approximated or twisted at this stage but only positioned in the proper orifices. Otherwise, it would be more difficult to place them after the sternum is put together.

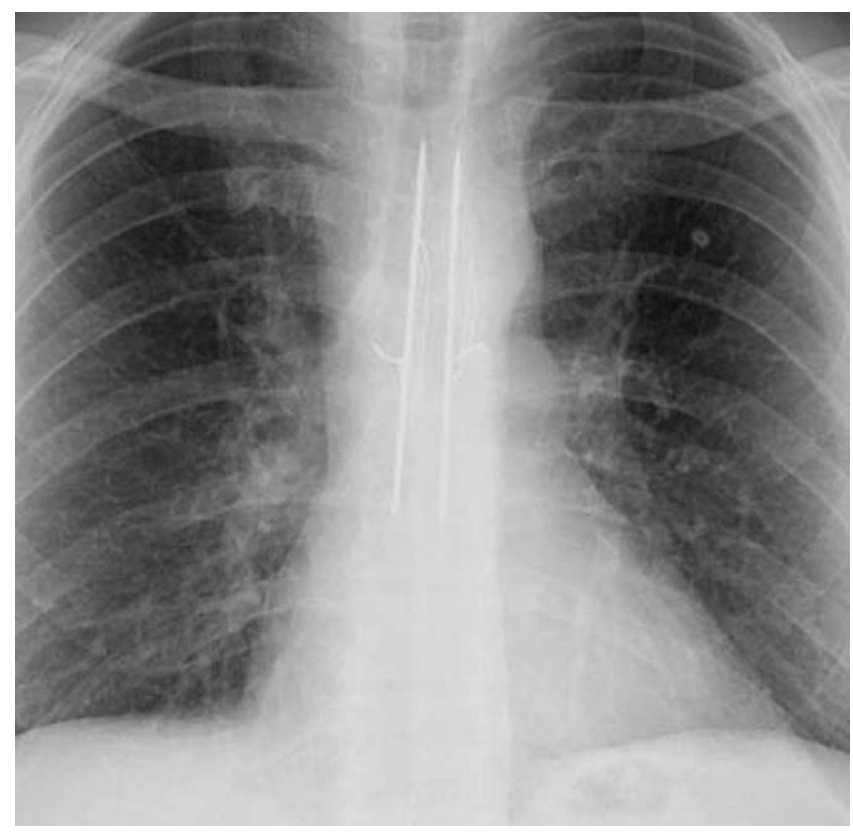

Figure 6. Postoperative view of a repaired sternal fracture with the pins in position, as well as the $\mathbf{2}$ parallel sternal wires. 
Next we insert 2 threaded Steinmann pins (3/32 inch or $1 / 16$ inch in diameter) in the distal body of the sternum, parallel to each other in a tangential manner. These are advanced within the thickness of the bone, crossing the line of fracture into the manubrium for at least 1 inch. Our method is similar to that described by Rydell and Jennings ${ }^{10}$ for the treatment of pectus excavatum. At the time the pins are advanced, the 2 fragments of the sternum must be set tight together, and no gap should be left between the 2 fragments. The pins are then cut flush to the sternum. The wires previously placed are pulled snugly together and twisted, burying the cut ends in the soft tissue lateral to the sternum (Figure 5). We then close the fascia layer (containing the pectoralis major muscles) and the skin incision. The patient is hospitalized for an average of 3 days, and total recovery time is 6 to 8 weeks.

\section{Follow-up}

Of our 12 patients, 2 had late minor complications. In 1 patient the Steinmann pin migrated distally and protruded under the skin, requiring pin removal. This complication occurred at the beginning of our experience, when we were using smooth plain pins, but since we switched to threaded pins, we have had no other problems.

In the other patient, a slender person, discomfort was caused by 1 of the twisted wires under the skin, and therefore we removed that wire. Figure 6 shows the postoperative radiographic aspect of the chest with the 2 intramedullary pins in position and the sternal wires at the level of the fracture.

Our current follow-up for all 12 patients extends from 6 months to 17 years, with no late complications and no other sequelae. All 12 patients are fully active and can use their arms without restriction.

\section{Conclusions}

Fractures of the sternum are not uncommon as a result of direct trauma to the sternum, particularly in car accidents, including steering wheel direct blows to the sternum, or severe falls. I believe that all such fractures must be assessed and evaluated carefully. Many of them will need to be repaired to prevent chronic pain, deformity of the chest, deformity of the sternum, and even kyphosis. Any patient with a sternal fracture must be carefully evaluated not only at the time of trauma but also weeks later, after the healing has taken place. It is significant that all of our 12 patients had overlapping of the sternal bone fragments. In no patient was the normal position of the sternum preserved. All of them were symptomatic and restricted in their physical activities. As long as overlap of the bone fragments exists, patients should probably undergo an operation to correct this abnormality. Most patients with sternal trauma are seen in the emergency department and do not require an orthopedic surgeon; instead, they should be seen by a thoracic surgeon. Our current technique is safe and very effective, and long-term results have been highly satisfactory. In addition, the incision is cosmetically acceptable. Another option to consider is the technique described by Bertin and associates, ${ }^{11}$ but we do not have experience with it. The entire operation is done in an extrapleural manner, with no need for intrapleural chest tubes.

\section{References}

1. Peek GJ, Firmin RK. Isolated sternal fracture: an audit of 10 years experience. Injury. 1995;26:385-8.

2. Otremski I, Wilde BR, Marsh JL, et al. Fracture of the sternum in motor vehicle accidents and its association with mediastinal injury. Injury. 1990;21:81-3.

3. Trinca GW, Doaley BJ. The effects of mandatory seat belt wearing on the mortality and pattern of injury of car occupants in motor vehicle crashes in Victoria. Med J Aust. 1975;1:675-8.

4. Potaris K, Gakidis J, Mihos P, et al. Management of sternal fractures: 239 cases. Asian Cardiovasc Thorac Ann. 2003;10:145-9.

5. Brookes JG, Dunn RJ, Rogers IR. Sternal fractures: a retrospective analysis of 272 cases. J Trauma. 1993;35:46-54.

6. Harley DP, Mena I. Cardiac and vascular sequelae of sternal fractures. J Trauma. 1986;26:553-7.

7. Sadaba JR, Oswal D, Musch CM. Management of isolated sternal fractures: determining the risk of blunt cardiac injury. Ann R Coll Surg Engl. 2000;82:162-6.

8. Gouldman JW, Miller RS. Sternal fracture: a benign entity? Am Surg. 1997;63:17-9.

9. Huggett J, Roszler MH. CT finding of sternal fracture. Injury. 1998; 29:623-6.

10. Rydell JR, Jennings WK. The surgical treatment of funnel chest deformity. Am J Surg. 1954;88:69-75.

11. Bertin KC, Rice RS, Doty DB, et al. Repair of transverse sternal nonunions using metal plates and autogenous bone graft. Ann Thorac Surg. 2003;73:1661-2. 